\title{
RISK PATTERNS AND CORRELATED BRAIN ACTIVITIES. MULTIDIMENSIONAL STATISTICAL ANALYSIS OF FMRI DATA IN ECONOMIC DECISION MAKING STUDY
}

\author{
ALENA VAN BÖMMEL
}

MAX PLANCK INSTITUTE FOR MOLECULAR GENETICS

SONG SONG

C.A.S.E. CENTER FOR APPLIED STATISTICS AND ECONOMICS, HUMBOLDT-UNIVERSITÄT ZU BERLIN

PIOTR MAJER

C.A.S.E. CENTER FOR APPLIED STATISTICS AND ECONOMICS, HUMBOLDT-UNIVERSITÄT ZU BERLIN

PETER N.C. MOHR

DEPARTMENT OF EDUCATION AND PSYCHOLOGY, FREIE UNIVERSITÄT BERLIN

\author{
HAUKE R. HEEKEREN
}

DEPARTMENT OF EDUCATION AND PSYCHOLOGY, FREIE UNIVERSITÄT BERLIN

\author{
WOLFGANG K. HÄRDLE
}

SCHOOL OF BUSINESS, SINGAPORE MANAGEMENT UNIVERSITY

\begin{abstract}
Decision making usually involves uncertainty and risk. Understanding which parts of the human brain are activated during decisions under risk and which neural processes underly (risky) investment decisions are important goals in neuroeconomics. Here, we analyze functional magnetic resonance imaging (fMRI) data on 17 subjects who were exposed to an investment decision task from Mohr, Biele, Krugel, Li, and Heekeren (in NeuroImage 49, 2556-2563, 2010b). We obtain a time series of three-dimensional images of the blood-oxygen-level dependent (BOLD) fMRI signals. We apply a panel version of the dynamic semiparametric factor model (DSFM) presented in Park, Mammen, Wolfgang, and Borak (in Journal of the American Statistical Association 104(485), 284-298, 2009) and identify task-related activations in space and dynamics in time. With the panel DSFM (PDSFM) we can capture the dynamic behavior of the specific brain regions common for all subjects and represent the high-dimensional time-series data in easily interpretable low-dimensional dynamic factors without large loss of variability. Further, we classify the risk attitudes of all subjects based on the estimated low-dimensional time series. Our classification analysis successfully confirms the estimated risk attitudes derived directly from subjects' decision behavior.
\end{abstract}

Key words: risk, risk attitude, fMRI, decision making, semiparametric model, factor structure, SVM.

\section{Introduction}

Decision making is a complex process of integrating and comparing various aspects of choice options. In the past years decision neuroscience has made important progress in ground-

Requests for reprints should be sent to Piotr Majer, C.A.S.E. Center for Applied Statistics and Economics, Humboldt-Universität zu Berlin, Berlin, Germany. E-mail: piotr.majer@ wiwi.hu-berlin.de 
ing these aspects of decision making in neural systems (Heekeren, Marrett, \& Ungerleider, 2008; Rangel, Camerer, \& Montague 2008). Economic decision making is usually described as a form of value-based decision making, in which individuals are assumed to first evaluate the different choice alternatives, then compare the different values, and finally choose the one with the highest value.

Various studies in decision neuroscience (Kable \& Glimcher, 2007; Plassmann, O’Doherty, $\&$ Rangel, 2007) investigated neural representations of value, the crucial metric in value-based decision models. Kable and Glimcher (2007) and Plassmann et al. (2007) indicate that the value of a choice alternative is represented in a brain network consisting of medial orbitofrontal cortex (mOFC), ventromedial prefrontal cortex (VMPFC), ventral striatum (VST), and posterior cingulate cortex (PCC). Although most models of decision making share the idea of a value metric that is determined and compared to make a decision, the assumed valuation process differs significantly from one model to another.

For decisions under risk a crucial metric that is assumed to influence the value of a choice alternative is the decision maker's risk attitude. In utility-based models of decision making under risk, the risk attitude determines the curvature of the utility function, thereby influencing the valuation process of risky choice alternatives. In contrast, risk-return models (Sarin \& Weber, 1993; Weber \& Johnson, 2009b), incorporate the risk attitude as a weighting factor on the risk metric of the model. So far, only a few studies have tried to identify neural representations of risk attitude or neural mechanisms reflecting the effect of the risk attitude on the valuation process. One study found correlations between risk attitude and risk-related brain activity in lateral orbitofrontal cortex (1OFC) for risk-averse individuals and in medial OFC for risk-seeking individuals (Tobler, O'Doherty, Dolan, \& Schultz, 2007). Another study (Mohr et al., 2010b) found that inter-individual differences in decision-related brain activity in IOFC and PCC correlated with inter-individual differences in risk attitudes independent of the current level of risk. The authors showed that the value signal in the ventrolateral prefrontal cortex (VLPFC) increased with risk in risk-seeking individuals and decreased with risk in risk-averse individuals, thereby reflecting the risk attitude.

All the above mentioned studies used the functional magnetic resonance imaging (fMRI) technique to detect brain regions activated by the stimulus. This technique measures local changes during neuronal activity in the blood flow, volume and oxygen level, called the bloodoxygen-level dependent (BOLD) signal. The conventional model for fMRI analysis is the general linear model (GLM). Although it is frequently used in neuroscience and has led to important insights into the neurobiological processes underlying cognition and emotion, the GLM approach has some important limitations. First, it focuses on task-related changes in the mean BOLD signal. Thereby, the GLM neglects information that might be carried by the variability of the BOLD signal (Mohr \& Nagel, 2010). For example, Samanez-Larkin, Kuhnen, Yoo, and Knutson (2010) found that the relationship between age and the number of risk-seeking mistakes in a risky decision making task was mediated by the temporal variability of the BOLD signal. Second, the GLM is a model-based approach and can, therefore, only detect effects that were previously hypothesized and modeled. Recent advances in model-free analysis techniques, such as the tensor probabilistic independent component analysis (T-PICA) developed by Beckmann and Smith (2005), have the potential to detect effects without any constraints on a priori hypotheses or modeling.

In line with these approaches we investigate individual differences in risk attitudes with a model-free technique focusing on the temporal variability of its components. Specifically, we use the dynamic semiparametric factor model (DSFM) introduced by Park et al. (2009) to reduce dimensions of the high-frequency, high-dimensional multi-subject fMRI data. DSFM estimates both spatial factors common for all studied subjects and subject-specific factor loadings varying in the temporal domain. 
The DSFM factorization technique is set up similar to the T-PICA approach (Beckmann \& Smith, 2005). T-PICA decomposes the full data set into factors in spatial, temporal and subject domains using a large number of model control variables and parameters. In contrast, the panel version of the DSFM finds spatial factors common for all subjects and time-varying factor loadings which include also the subject specificity. All easily interpretable components are estimated by using a small number of model parameters. In summary, the parsimoniousness and flexibility of DSFM allows us to capture the variability of corresponding brain regions by subject-specific time-series loadings. Further, we hypothesize that the temporal variability of components corresponding to factors in brain regions related to value processing (e.g., mOFC and PC) is correlated with the risk attitude of individuals.

In the next section, we describe the experimental procedures, the PDSFM methodology and the risk attitude estimation procedure. In Section 3, we describe the model parameters, the estimated spatial factors with time-varying factor loadings and the risk classification based on the PDSFM estimates. We conclude with a summary and discussion section.

\section{Materials and Methods}

\subsection{Experimental Procedures}

We reanalyzed data from a previously published study (Mohr et al., 2010b) with 22 young volunteers (age 18-35 years, 11 females). All participants were native German speakers, righthanded and had no history of neurological or psychiatric diseases. Three participants had to be excluded due to extensive head motion ( $>5 \mathrm{~mm}$ absolute head movement) or modeling problems (always chose only one alternative). Another two were excluded due to a different scanning frequency.

To simulate decision making under risk, participants in the study fulfilled a Risk Perception and Investment Decision (RPID) task. Each trial of the RPID consisted of two phases: the presentation of a return stream, followed by a decision or subjective judgment task (see Figure 1). In investment situations investors are often confronted with past performance data of possible investments. To mimic this situation, in the first phase a stream of 10 returns from an investment was sequentially presented for $2 \mathrm{~s}$. These 10 returns provided information about the past performance of a given investment. In the experiment, each return stream was independent of the others, drawn from Gaussian distribution with varying means $(6 \%, 9 \%$, and $12 \%)$ and standard deviations $(1 \%, 5 \%$, and $9 \%)$, resulting in nine different combinations of means and standard deviations. In the second phase, subjects performed one of three possible tasks in each trial (each $7 \mathrm{~s}$ ) without knowing in advance which one they would have to perform after the stream.

Three tasks were used to investigate choices as well as perceived risk and subjective expected return, as specified in recent psychological risk-return models (Weber \& Johnson, 2009a). In the decision task the subjects had to make a choice between an investment with a $5 \%$ fixed return (safe investment) and the investment represented by the return stream just presented (risky investment). In the other two tasks subjects reported their subjective expected return (on a scale from $-5 \%$ to $+15 \%$ ) and perceived risk (from $0=$ no risk to $100=$ maximum risk) of the investment represented by the return stream. Subjects performed each task (decision, subjective expected return, perceived risk) 27 times resulting in 81 trials in total.

Subjects received a flat payment of 10 Euros for their use in the experiment and a virtual endowment of 100 Euros to invest. The participants were instructed that after the experiment, one of their 27 choices will be randomly chosen to determine decision dependent payments. If the subject would choose the safe option in the respective trial, she would get 5 Euros $(5 \%$ of 100 Euros) in addition to the 10 Euros. If a subject would choose the risky option in this trial, a random return was drawn from a Gaussian distribution with the same mean and standard 


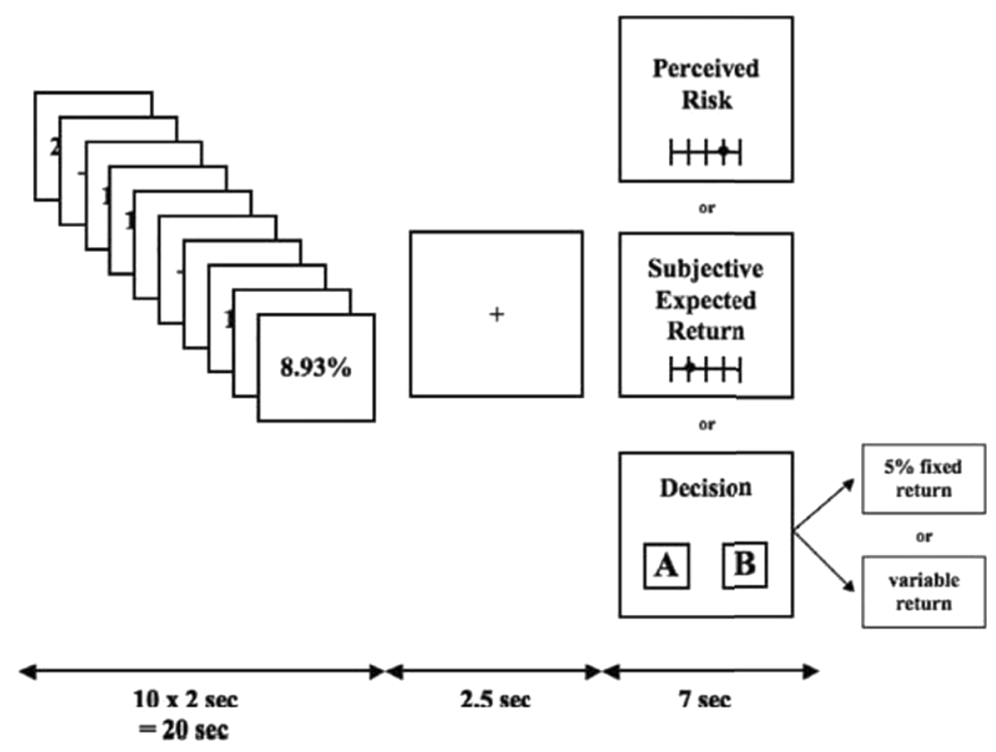

FIGURE 1 .

RPID task: Subjects were presented with streams of 10 returns from an investment. Then, they either (a) judged the subjective expected return of the return stream, (b) judged the perceived risk of the return stream, or (c) chose between an investment with a fixed return of $5 \%$ and an investment with a variable return which was represented by the return stream (modified from Mohr et al., 2010b).

deviation as the respective return stream. The resulting outcome (return times 100 Euro) was added to or subtracted from the flat payment.

\section{2. fMRI Data}

fMRI data were acquired on a 1.5 T Magnetom Sonata fMRI system (Siemens, Erlangen, Germany) equipped with a standard head coil. We used a vacuum pad to minimize head motion. Functional images were acquired using a BOLD-sensitive T2*-weighted echo-planar imaging (EPI) sequence [time resolution (TR), $2500 \mathrm{~ms}$; echo time (TE), $40 \mathrm{~ms}$; flip angle, $90^{\circ}$; field of view, $256 \mathrm{~mm}$; matrix, $64 \times 64 \mathrm{~mm}$; 26 axial slices approximately parallel to the bicommissural plane; slice thickness, $4 \mathrm{~mm}]$.

The data was initially pre-processed with FSL 4.0 (FMRIB's Software Library, http://www. fmrib.ox.ac.uk/fsl/). Pre-processing included motion correction and slice-time correction. Additionally, images were normalized into a standard stereotaxic space (Montreal Neurological Institute (MNI), Montreal, Quebec, Canada).

\subsection{Panel Dynamic Semiparametric Factor Model}

To capture the temporal variability in the high-dimensional fMRI series, which may be related to individual differences in risk attitude, we use a dynamic semiparametric factor model (DSFM) which was proposed by Park et al. (2009). DSFM is a model-free multivariate method that is able to identify activated brain regions (factors) and corresponding low-dimensional time series (factor loadings) in only one estimation step (Park et al., 2009). In addition, the panel version of the DSFM allows determining active brain areas common for multiple subjects, whereas the individual changes are described by subject-specific time series.

From a statistical point of view, the BOLD signal of all voxels during the whole experiment can be considered as a multidimensional time series. The following DSFM is calibrated to study such high-dimensional time series: 


$$
\begin{aligned}
Y_{t, j} & =m_{0}\left(X_{t, j}\right)+\sum_{l=1}^{L} Z_{t, l} m_{l}\left(X_{t, j}\right)+\varepsilon_{t, j}, \quad 1 \leq j \leq J, 1 \leq t \leq T . \\
& \stackrel{\text { def }}{=} Z_{t}^{\top} m\left(X_{t, j}\right)+\varepsilon_{t, j}=Z_{t}^{\top} A^{*} \Psi_{t, j}+\varepsilon_{t, j} .
\end{aligned}
$$

Here, $Z_{t}=\left(\mathbf{1}, Z_{t, 1}, \ldots, Z_{t, L}\right)^{\top}$ is an unobservable $(L+1)$-dimensional stochastic process and $m$ is an $(L+1)$-tuple $\left(m_{0}, \ldots, m_{L}\right)$ of unknown real-valued functions $m_{l}$. The predictors $X_{t, j} \in \mathbb{R}^{d}$ are known, and $Y_{t, j} \in \mathbb{R}$ is the response variable of interest (the BOLD signal). The errors $\varepsilon_{t, j}$ are assumed to be independent of $Z_{t, j}$ and have zero means and finite second moments. The functions $m_{l}$ are approximated by a space basis $\Psi_{t, j}=$ $\left[\psi_{1}\left(X_{t, j}\right), \ldots, \psi_{K}\left(X_{t, j}\right)\right]^{\top}$ and corresponding $(L+1) \times K$ matrix of unknown coefficients $A^{*}$. More precisely, $\left[\psi_{1}\left(X_{t, j}\right), \ldots, \psi_{K}\left(X_{t, j}\right)\right]^{\top}$ denote quadratic tensor B-splines on $K$ equidistant knots. The estimates of $Z_{t}^{\top}$ and $A^{*}$ are found by minimizing

$$
S\left(\widehat{Z}_{t}, \widehat{A}^{*}\right)=\arg \min _{Z_{t}, A^{*}} \sum_{t=1}^{T} \sum_{j=1}^{J}\left\{Y_{t, j}-Z_{t}^{\top} A^{*} \Psi_{t, j}\right\}^{2} .
$$

The minimum is found by the Newton-Raphson algorithm. The parametric part $\widehat{Z}_{t}$ captures the evolution in time, while $\widehat{A^{*}}$ represents the smooth, nonparametrically estimated spatial structure.

In our experiment, the voxel's index $\left(i_{1}, i_{2}, i_{3}\right)$ is the covariate $X_{t, j}$, and the normalized BOLD signal is the dependent variable $Y_{t, j} ; j=1, \ldots, J ; t=1, \ldots, T$. Due to the computational limitations (software memory capacity), we used the first part (first 722 observations including 15 out of 27 trials) of the experiment for our analysis. To further simplify numerical operations, blank areas were removed from the original data. Thus, the spatial and temporal dimensions are given by $J=91 \times 92 \times 71=594412$ and $T=722$, respectively. Note that the covariate is time-invariant, and it follows that $X_{t, j}=X_{j}=\left(i_{1}, i_{2}, i_{3}\right) \in\{(1 ; 91),(1 ; 92),(1 ; 71)\}$.

Since we want to analyze all tested subjects $i=1, \ldots, I$ in one model to estimate the common spatial regions responding to the stimulus, we extended (1) to a panel dynamic semiparametric factor model (PDSFM),

$$
\begin{gathered}
Y_{t, j}^{i}=m_{0}\left(X_{j}\right)+\sum_{l=1}^{L}\left(\bar{Z}_{t, l}+\alpha_{t, l}^{i}\right) m_{l}\left(X_{j}\right)+\varepsilon_{t, j}^{i}, \\
1 \leq j \leq J, 1 \leq t \leq T, 1 \leq i \leq I
\end{gathered}
$$

$\alpha_{t, l}^{i}$ is the (random) individual effect for subject $i$ on function $m_{l}$ at time point $t$. For identification purposes with respect to subjects, we assume that expectation of the individual effects over all subjects and over all functions $m_{l}$ sums to zero, i.e.,

$$
\mathrm{E}\left[\sum_{i=1}^{I}\left(\sum_{l=1}^{L} \alpha_{t, l}^{i} m_{l}\left(X_{j}\right) \mid X_{j}\right)\right]=0 .
$$

It is reasonable to claim that different subjects have different patterns of brain activation to external stimuli which can be characterized by different stochastic processes $Z_{t}^{i}$. On the other hand, we can assume that the subjects share essentially the same spatial structure of the brain which could be characterized by identical $m_{l}$ functions. Our analysis concentrates on the detection of these common active brain regions over all subjects. Hereinafter, we assume that these regions are homogeneous for all individuals and, therefore, can be modeled by a joint (average) spatial factor denoted as $\bar{m}_{l}, l=1, \ldots, L$. The activation differences between the individuals are captured by their specific low-dimensional time series $Z_{t, l}^{i}$. 
For the averaged fMRI series, $\bar{Y}_{t, j}$ obeys an equivalent relation to (1):

$$
\bar{Y}_{t, j}=\bar{m}_{0}\left(X_{j}\right)+\sum_{l=1}^{L} \bar{Z}_{t, l} \bar{m}_{l}\left(X_{j}\right)+\varepsilon_{t, j}, \quad 1 \leq j \leq J, 1 \leq t \leq T,
$$

with factor loadings $\bar{Z}_{t, l}$ corresponding to common factors $\bar{m}_{l}$.

After the spatial functions are determined, the subject-specific time series $Z_{t, l}^{i}=\bar{Z}_{t, l}+\alpha_{t, l}^{i}$ can be estimated using the ordinary least square method from the following equation:

$$
Y_{t, j}^{i}=\bar{m}_{0}\left(X_{j}\right)+\sum_{l=1}^{L} Z_{t, l}^{i} \bar{m}_{l}\left(X_{j}\right)+\varepsilon_{t, j}^{i} .
$$

The statistical inference of the whole system is then based on the low-dimensional time-series analysis for $Z_{t, l}^{i}, i=1, \ldots, I$. Park et al. (2009) has shown that the difference between the inference based on the estimated low-dimensional time series and the "true" unobserved time series is asymptotically negligible.

Our multi-subject multivariate estimation procedure can be summarized in the following steps:

1. Take the average $\bar{Y}_{t, j}$ of $Y_{t, j}^{i}$ across all subjects $i \in\{1,2, \ldots, I\}$ and estimate the common spatial factors $\widehat{\bar{m}}_{l}\left(X_{j}\right)$, as in the original DSFM approach (1). For the $\widehat{A^{*}} \Psi_{t, j}, \widehat{A^{*}}$ is obtained using the same Newton-Raphson algorithm based on (1) with the only difference that we do not use the original observations $Y_{t, j}$, but the averaged $\bar{Y}_{t, j}$ over different individuals.

2. Given the common $\bar{m}_{l}\left(X_{j}\right)$, estimate the subject-specific temporal factor loadings $\widehat{Z}_{t, l}^{i}$ from (5). Repeat this estimation procedure for all $i=1, \ldots, I$.

3. Analyze the joint factors $\bar{m}_{l}\left(X_{j}\right)$. Significant active brain regions are defined by the threshold of $0.5 \%$ - and $99.5 \%$-quantiles of the empirical distribution function of $\bar{m}_{l}\left(X_{j}\right)$ in all voxels.

4. Find the variations between individuals by looking at the factor loadings $\widehat{Z}_{t, l}^{i}$. Is it possible to reconstruct subjects' behavior just by analyzing this low-dimensional time series?

\subsection{Behavioral Modeling}

According to subjects' responses, the risk attitude for each individual could be estimated. We applied the following psychological risk-return model (detailed description can be found in Mohr et al., 2010b): Importantly, behavioral research has shown that subjective expected return and perceived risk can vary significantly depending on the context of the decision, challenging the predictive power of the objective measures expected return and variance of returns (Weber and Milliman, 1997; Weber, Siebenmorgen, \& Weber, 2005).

$$
V_{i}(x)=\mu_{i}(x)-\phi_{i} \sigma_{i}(x) .
$$

In this equation $V_{i}(x)$ defines the value a subject $i$ assigns to an investment $x, \mu_{i}(x)$ represents the subjective expected return, $\sigma_{i}(x)$ represents the subject's perceived risk, and $\phi_{i}$ is the individual risk weight.

For each subject, the best individual model for subjective expected return and perceived risk was estimated using the leave-one-out cross-validation method on the 27 trials. A total of five different models for subjective expected return, namely, mean, recency, primacy, overweight $<0 \%$, and overweight $<5 \%$ and six models for the perceived risk, namely, standard deviation, coefficient of variation, probability $<0 \%$, probability $<5 \%$, range, and coefficient of range, were compared. The individual risk weight $\phi_{i}$ was estimated by logistic regression using the best individual models for $\mu_{i}(x)$ and $\sigma_{i}(x)$. 
TABLE 1.

Explained variation in percent for the model with different numbers of factors $L$. The explained variation is averaged over all 17 analyzed subjects.

\begin{tabular}{lcccc}
\hline$L=2$ & $L=4$ & $L=5$ & $L=10$ & $L=20$ \\
\hline 92.07 & 92.25 & 92.29 & 93.66 & 95.19 \\
\hline
\end{tabular}

The risk attitude can then be measured as value reduction in Euros for maximum risk (the case when the subjective perceived risk $=100$ ) compared to minimal risk (the case when the return $=5 \%$ ). All subjects were classified as risk averse indicated by a positive risk weight. However, for seven subjects the risk attitude was low (risk weight $\leq 5$ ) resulting in only a small influence of risk on value. Following the classification in Mohr et al. (2010b), the seven subjects with the lowest risk attitude were classified as weakly risk averse. All subjects with risk weight $>5$ were classified as strongly risk averse. A detailed description of the estimation procedure can be found in Mohr et al. (2010b).

\section{Results}

In this section we describe the choice of the model parameters and the computational time for the studied data set. Further, we discuss a selection of estimated factors and corresponding factor loadings for selected subjects. The classification procedure described in the last paragraph is based only on the fMRI data and predicts the subjects' risk aversion with high precision.

\subsection{Computational Time}

The analyzed raw data are high dimensional $(722 \times 594412=429165464)$ and require large memory $(17 \times 1.3 \mathrm{~GB})$. On our server equipped with $2.8 \mathrm{GHz}$ CPU and $16 \mathrm{~GB}$ RAM memory loading, the data loading takes around 1 hour. Each step in the Newton-Raphson optimization in Equation (2) lasts around 1 hour, and approximately 15 steps are needed for convergence (depending on the model setup). Therefore, the optimization of the model parameters is limited. After a preliminary analysis (data not shown) we chose quadratic tensor B-splines on equidistant knots as the space basis $\Psi_{j}$. We found that the increase in the number of knots provides better fit, but extends the computational time (quadratic growth). Thus, the number of knots in the three directions were set to 14,14 and 12 , which is a trade-off between the best possible resolution and a reasonable computational time. With this parameter choice, $250=(91 \times 92 \times 71) /(14 \times$ $14 \times 12$ ) points correspond to one three-dimensional quadratic B-spline basis function. The total computational time is then 22 hours.

\subsection{Model Parameters}

The most important parameter in our model is the number of factors $m_{l}$ as well as the number of corresponding factor loadings $Z_{t, l}$, denoted by $L ; l=1, \ldots, L$. It is worth noting here that the increase of $L$ does not extend significantly the computational time (linear growth). The choice of $L$ is based on both the specificity of factors' interpretation and the averaged explained variance by factors, which is given by

$$
E V(L)=1-\frac{\sum_{t=1}^{T} \sum_{j=1}^{J}\left\{Y_{t, j}-\sum_{l=0}^{L} Z_{t, l} m_{l}\left(X_{t, j}\right)\right\}^{2}}{\sum_{t=1}^{T} \sum_{j=1}^{J}\left\{Y_{t, j}-\bar{Y}\right\}^{2}} .
$$

Table 1 shows the averaged explained variation for different numbers of factors. Here, only slight differences between the explained variation for $L \geq 2$ can be observed. The fMRI signals 
$Y_{t, j}$ were explained mostly by the null-factor and the first two factors: $\widehat{m}_{0}\left(X_{t, j}\right)+Z_{t, 1} \widehat{m}_{1}\left(X_{t, j}\right)+$ $Z_{t, 2} \widehat{m}_{2}\left(X_{t, j}\right)$. The relatively small effects of the functions $m_{l}$ for $l \geq 2$ are due to the small differences of activation in the selected regions. The inclusion of a greater number of factors leads to detection of the important areas for decision making under risk. We choose $L=20$, which allows for relatively low complexity and an explicit interpretation of the functions $\widehat{\bar{m}}_{l}$. Residual analysis, derived from the model with $L=20$, are shown in Appendix A in Figures 6, 7 and 8 .

\subsection{Factors $\widehat{m}_{l}$}

In our study we are looking for spatial maps which undergo significant changes in BOLD signal during the experiment. These spatial maps (factors) determine the brain regions while the time evolution and subject specificity are captured by the corresponding factor loadings $Z_{t, l}^{i}$. After applying the PDSFM technique, we estimated 20 spatial factors. Six of them $\left(\widehat{m}_{l}\right.$, $l=5,9,12,16,17,18)$ correspond to brain areas mOFC and Parietal Cortex (PC) which were already found in decision making contexts (see Heekeren et al., 2008; Rangel et al., 2008 for a review). Figure 2 shows voxels with the largest values $\widehat{m}_{l}\left(X_{j}\right) \geq 99.95 \%$ of the empirical quantiles for these six factors in white. Factors $\widehat{m}_{5}, \widehat{m}_{12}, \widehat{m}_{16}, \widehat{m}_{17}$ and $\widehat{m}_{18}$ have the largest values in the mOFC, located in the bottom frontal part of the brain. Plassmann et al. (2007) showed that mOFC is associated with the value of a choice option. In other words, these five factors, $\widehat{m}_{5}$, $\widehat{m}_{12}, \widehat{m}_{16}, \widehat{m}_{17}$ and $\widehat{m}_{18}$, describe different areas in the mOFC, which are exposed to the largest activation changes during the experiment. Factors $\widehat{m}_{5}, \widehat{m}_{16}, \widehat{m}_{17}$ and $\widehat{m}_{18}$ differ in their vertical positions (as shown in Figure 2(a), (c)-(e) in the far left graphics), where factors $\widehat{m}_{5}$ and $\widehat{m}_{16}$ cover different vertical parts. This decomposition is possible due to the high resolution scanning analyzed here and deserves further research. Factor $\widehat{m}_{9}$ represents the PC situated at the intersection of visual, auditory, and tactile cortices which play a crucial role in selective attention (Behrmann, Geng, \& Shomstein, 2004). Beside these interesting factors connected with decision making, we detected other spatial maps that correspond to brain areas previously associated with motoric responses and visual perception. These maps are likely unrelated to the decision making process within the task but confirming the activity of regions which were necessary to provide the answer by pushing a button.

Herewith, we have shown that the estimated function $\widehat{m}_{l}, l=1, \ldots, L$ in our model represent those brain regions which were expected to be involved during the experiment (visualization, motoric, and value-of-choice areas). The activity of these regions changes over the subjects and with time. This variability is described by the subject's specific factor loadings $Z_{t, l}^{i}$ which are discussed in the next session.

\subsection{Factor Loadings $\widehat{Z}_{t, l}$}

The dynamics and subject specificity are jointly represented by the low-dimensional time series $\widehat{Z}_{t, l}^{i}, i=1, \ldots, I ; l=1, \ldots, L$. These subject-specific $\widehat{Z}_{t, l}^{i}$ correspond to the individual temporal differences of the activated brain regions in $\widehat{m}_{l}$. For better illustration we have selected two extreme subjects: 12 (with the smallest risk attitude) and 19 (with the largest risk attitude). Both shown time series $\widehat{Z}_{t, l}^{12}$ and $\widehat{Z}_{t, l}^{19}$ for $l=12,18$ show a high fluctuation around their mean value (Figure 3).

To investigate the activity behavior of the risk-related factors, we studied the corresponding factor loadings in the time point of risky decision and in the following three observations. In particular, to visualize the activities after the stimulus, we plotted the sum over all subjects of the normalized mean differences of these factor loadings (see the Figure 9 in Appendix B). Whereas some of the loadings tend to react positively to the stimulus $\left(Z_{5}\right.$ and $\left.Z_{18}\right)$, other loadings show a positive-negative fluctuation pattern $\left(Z_{12}, Z_{18}\right.$, and $\left.Z_{9}\right)$. Understanding those differences for 
(a)
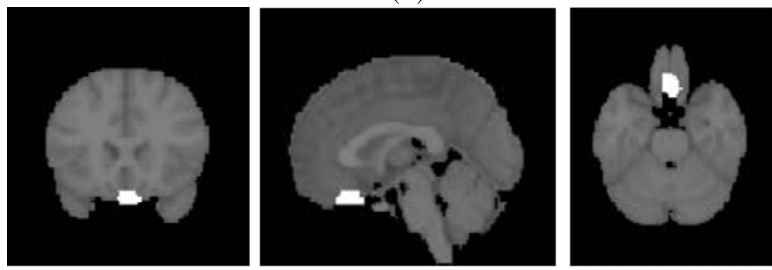

(b)
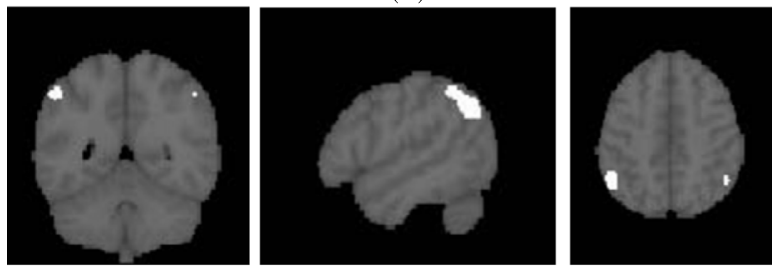

(c)
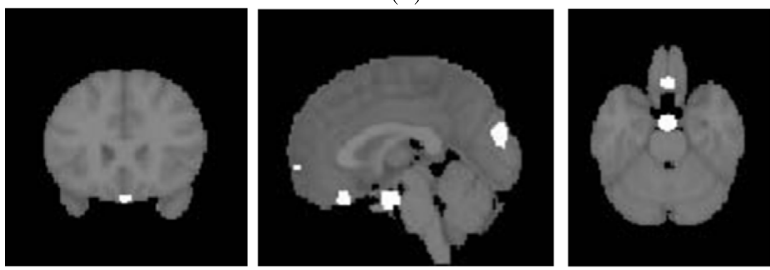

(d)
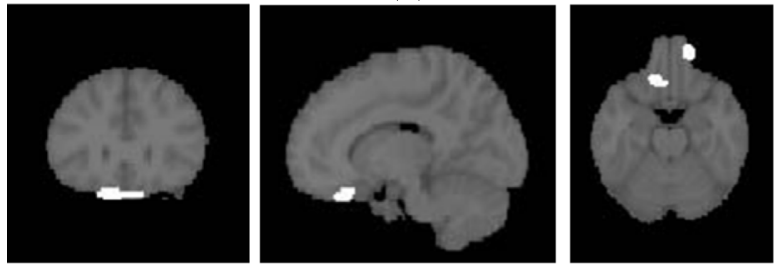

(e)
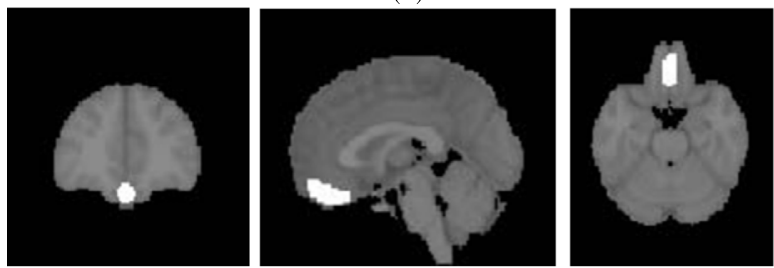

(f)
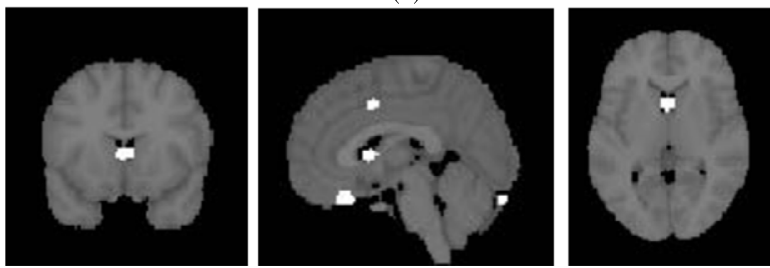

FIGURE 2.

Selected estimated functions $\widehat{m}_{l}, l=0, \ldots, L$ with $L=20$. (a) Estimated function $\widehat{m}_{5}$ with largest values in medial orbitofrontal cortex (mOFC); (b) $\widehat{m}_{9}$ with largest values in parietal cortex (PC); (c) $\widehat{m}_{12}$ with largest values in mOFC; (d) $\widehat{m}_{16}$ with largest values in mOFC; (e) $\widehat{m}_{17}$ with largest values in mOFC; (f) $\widehat{m}_{18}$ with largest values in mOFC. 

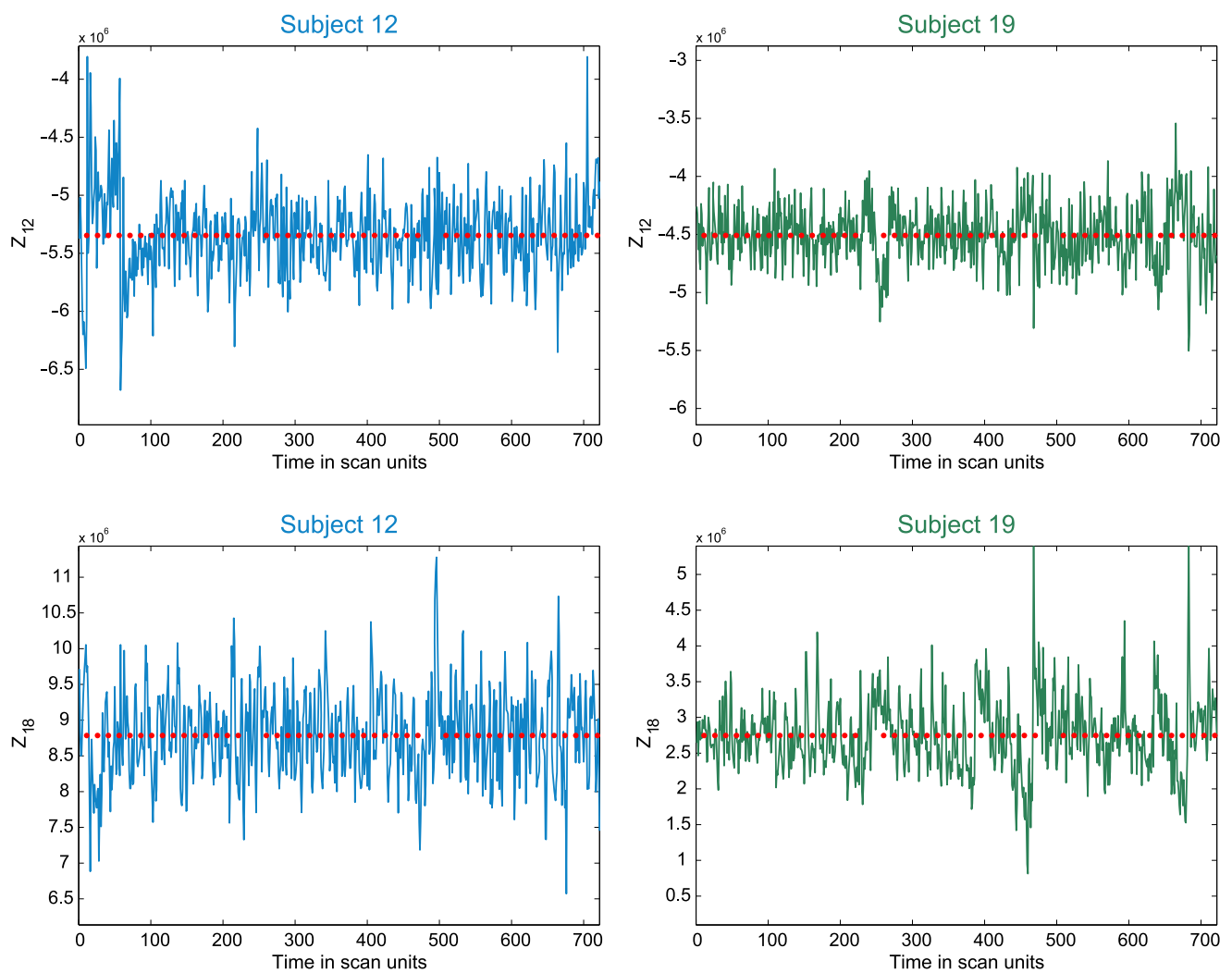

FIGURE 3

Factor loadings $\widehat{Z}_{t, 12}$ (top) and $\widehat{Z}_{t, 18}$ (bottom) for weakly risk-averse Subject 12 (blue line, left panels) and for strongly risk-averse Subject 19 (green line, right panels) during the experiment (722 time points). Red points correspond to the time points of stimuli. (Color figure online.)

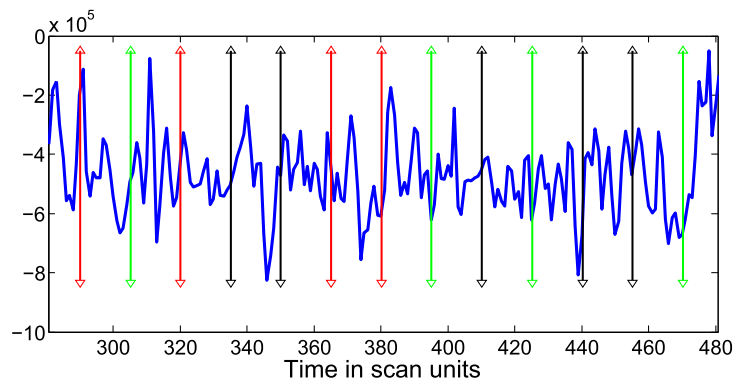

FIGURE 4.

Detailed view of factor loading $\widehat{Z}_{t, 1}$ for Subject 12 (blue line) with vertical lines at time points of stimuli of three different tasks: decision (red), subjective expected return (green) and perceived risk (black). (Color figure online.)

different mOFC parts picked up in the model goes beyond the scope of this paper and deserves further research. A better insight into the dynamics of the time series can be achieved while looking at the responses after the stimuli only and separating the subjects into the weakly and strongly risk averse. For an easier interpretation, first a detailed view of $\widehat{Z}_{t, 12}^{12}$ in 200 (out of 722) data points with three highlighted different stimulus types (i.e., decision, subjective expected return, perceived risk) is shown in Figure 4. The most important values of the factor loading 

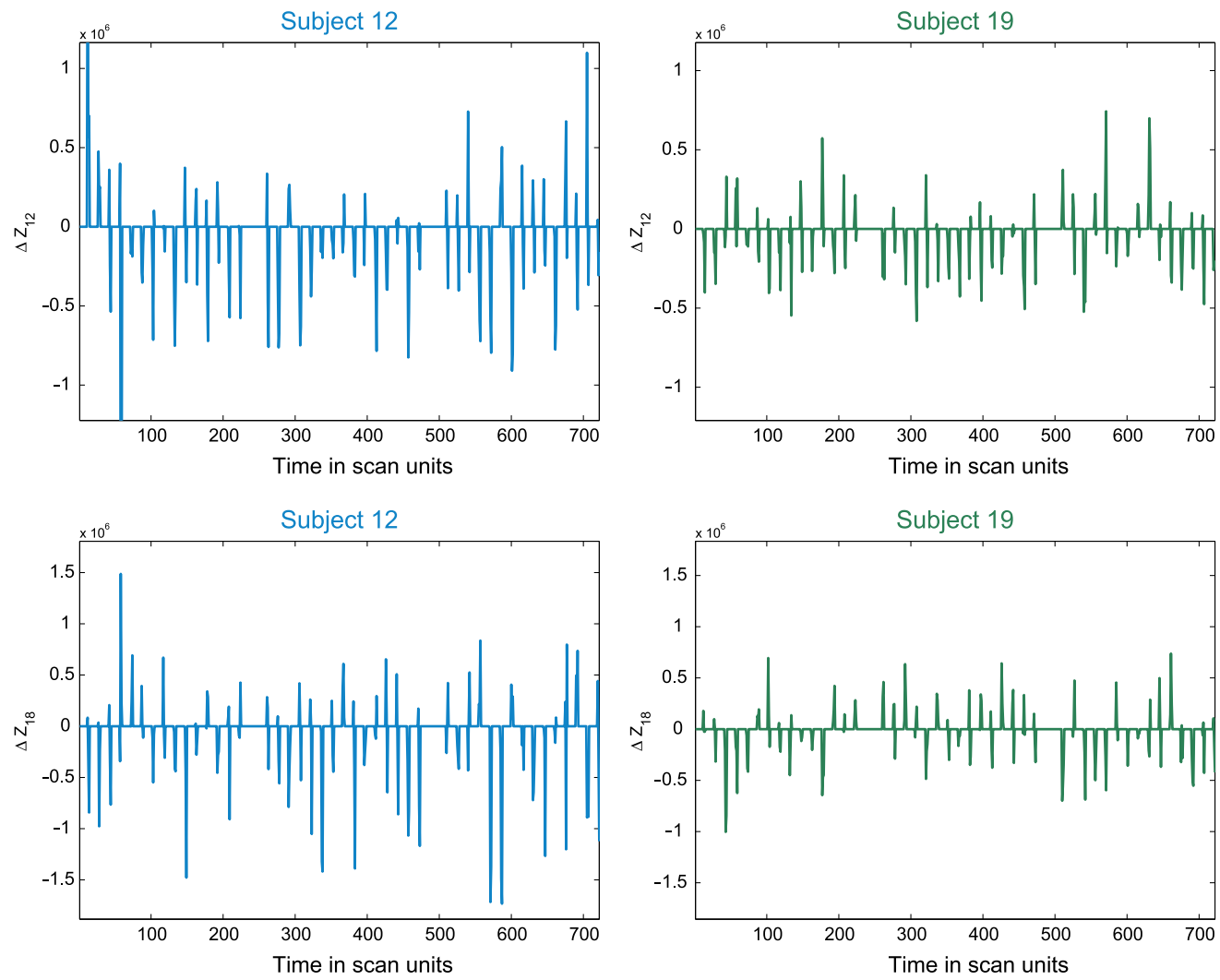

FIGURE 5 .

Reaction to stimulus for factor loadings $\widehat{Z}_{t, 12}$ (top) and $\widehat{Z}_{t, 18}$ (bottom) for weakly risk-averse Subject 12 (blue line, left panels) and strongly risk-averse Subject 19 (green line, right panels) during the whole experiment (45 stimuli). (Color figure online.)

$Z_{t, l}$ are the next three observations after each stimulus, measured up to 7.5 seconds after each stimulus, as the hemodynamic response has its peak usually around 6 seconds after the stimulus onset. Figure 5 shows these responses for studied factor loadings and representative subjects. Formally, we plotted $\Delta \widehat{Z}_{s, l} \stackrel{\text { def }}{=} \widehat{Z}_{s+\tau, l}-\widehat{Z}_{s, l}$ against $t$, where $s$ is the time when a stimulus was given and $\tau=1,2,3$ are the next three observations. In Figure 5, one can observe that the responses to the stimulus of the weakly risk-averse individual show a significantly different volatility than the responses of the strongly risk-averse individual. We found this volatility pattern in all factor loadings corresponding to the selected factors, namely, for $l=5,9,12,16,17,18$ (see Appendix C, Figure 10).

\subsection{Risk Classification}

Now that we have reasonably interpreted the estimated factors $\widehat{\bar{m}}_{l}$ and have described the behavior of the factor loadings $\widehat{Z}_{t, l}^{i}$, we next try to connect our findings with the risk attitude of a subject. Without knowing the subjects' answers, based only on a low-dimensional $\widehat{Z}_{t, l}^{i}$ representation of BOLD signal, we develop a classification method which can predict the risk attitude. For this purpose we use the functions $\Delta \widehat{Z}_{t, l}^{i}, l=5,9,12,16,17,18$ and $i=1,2, \ldots, I$, since they correspond to the brain activity of factors, which are linked with utility and decisions under risk.

As described in Section 2, there were three different types of answers which subjects had to make. Since risk attitude influences the value of a choice alternative, we considered $\Delta \widehat{Z}_{t, l}^{i}$, 
TABLE 2 .

Classification rates of the SVM method using standard deviation (left) and mean (right) of the $\Delta \widehat{Z}_{t, j}, j=$ $5,9,12,16,17,18$.

\begin{tabular}{lccccccc}
\hline STD & \multicolumn{2}{c}{ Estimated } & MEAN & \multicolumn{2}{c}{ Estimated } \\
\hline \multirow{4}{*}{ Data } & Strongly & Weakly & & & Strongly & Weakly \\
& Weakly & 0.97 & 0.03 & \multirow{2}{*}{ Data } & Strongly & 0.70 & 0.30 \\
& 0.25 & 0.75 & & Weakly & 0.55 & 0.45 \\
\hline
\end{tabular}

$l=5,9,12,16,17,18$ only after the decision task (where subjects decided between a risky or safe investment). Further, we use only the three observation points after the stimulus in the classification analysis (see paragraph Factor loadings $\widehat{Z}_{t, l}$ ). Since the observations are not exactly at the time points of the BOLD peak and we want to capture the variability in the stimulus responses, the average value of the responses after each of the 15 decision exercises is calculated: $\bar{\Delta} \widehat{Z}_{s, l}^{i}=\frac{1}{3} \sum_{\tau=1}^{3} \Delta \widehat{Z}_{s+\tau, l}^{i}, l=5,9,12,16,17,18$. Here, $\bar{\Delta} \widehat{Z}_{s, l}^{i}$ denotes the average reaction to the decision stimulus after the task $s$, for loading $l$ and subject $i$. Further, we calculated the variance of $\bar{\Delta} \widehat{Z}_{s, l}^{i}$ and observed higher variability for weakly risk-averse subjects than for strongly averse subjects (see Appendix D, Figures 11-16). In order to confirm that finding, these six variables, corresponding to $l=5,9,12,16,17,18$, were chosen as the input variables for the classification algorithm.

In the next step, we classify studied subjects based on the standard deviation of the data extracted from the BOLD signal. Classification analysis of the subjects was conducted via Support Vector Machines (SVM), (Cortes \& Vapnik, 2005). In the learning step, strongly risk-averse subjects were labeled with 1 and weakly risk-averse subjects with -1 . The estimated risk attitude described in Behavioral Modeling were used for the validation of the classification predictions. In order to avoid overparametrization and overly optimistic results, we applied the double crossvalidation method to tune the SVM and estimate the classification rate. The algorithm can be described as follows

1. Separate the data into a training set and a test set (leave-one-out procedure).

2. Apply the leave-one-out cross-validation method on the training set only and tune the algorithm.

3. Classify the test data.

4. Repeat Steps 1-3 for all the different test sets.

5. Average the classification rate over all iterations in Step 4.

Using the standard deviation of the differences and the optimal SVM parameters, the classification rate was $97 \%$ for strongly risk-averse individuals and $75 \%$ for weakly risk-averse individuals (Table 2). We compare our classification performance with an SVM classifier, taking as input variables the means of the averaged reaction to the decision stimulus $\bar{\Delta} \widehat{Z}_{t, l}$. Table 2 clearly shows that the mean of the averaged reaction does not reflect the differences between weakly and strongly risk-averse subjects. These two classes can be distinctly identified by their volatility. Thus, the risk attitude of a subject is derived directly from the time series $\widehat{Z}_{t, l}^{i}, l=5,9,12,16,17,18$ - the low-dimensional representation of the BOLD signals series. The class imbalancing in this two-step procedure ranges from $33 \%$ (10 vs. 5) up to $47 \%$ (8 vs. 7) and should not lead to a significant bias of the classifier, which is confirmed by high, averaged classification rates.

We have provided the SVM classification using a wide range of prior parameters to the Gaussian kernel, the capacity $C$ and the radial basis coefficient $r$. Table 3 summarizes the results obtained by using different values of these parameters. The classification rates are the averages over the parameter ranges. 
TABLE 3.

Classification rates of the SVM method using different values of parameters $r$ and $C$ for mean and standard deviation.

\begin{tabular}{lccc}
\hline & Rate & $r$ & $C$ \\
\hline Std & 0.81 & $0.6-1.00$ & $1-80$ \\
Mean & 0.60 & $0.02-1.00$ & $1-80$ \\
\hline
\end{tabular}

TABLE 4.

Classification rates of the SVM method using different values of parameters $L$ for standard deviation.

\begin{tabular}{lcccc}
\hline$L=2$ & $L=4$ & $L=5$ & $L=10$ & $L=20$ \\
\hline- & 61.40 & 58.82 & 58.82 & 87.13 \\
\hline
\end{tabular}

\subsection{Classification Results for a Different Model Specification L}

We analyzed the robustness of the classification results with respect to the parameter $L$ defining the number of factors. For the case $L=2$, none of the two factors $\widehat{m_{l}}$ represents the value-of-choice areas. The model specification with $L=4$ detects two factors with the highest values in the mOFC. In the model with $L=5$, three factors are attributed to the value-of-choice areas (mOFC). For the model specification with $L=10$ we find five factors with the highest values in the mOFC and Amygdala. In the next step the classification analysis, as described in the previous paragraph, was done for all different setups. The classification results are summarized in Table 4 . We can conclude our findings as follows. The inclusion of a larger number of factors $(2<$ $L \leq 10$ ) does not necessarily increase the classification performance. Models with a relatively low number of factors $(L \leq 10)$ do not reveal a good classification performance implying higher order models are needed to capture the dynamics of the relevant brain regions and to achieve a reasonable classification rate (at the cost of parsimony of the model and the computational time).

\section{Discussion}

Decision making is a complex process consisting of valuation, comparison, and the final choice. Decision neuroscience has frequently investigated neural representations of value, the key metric in the decision models. Previous studies showed that the BOLD response in VMPFC, PCC, VST, mOFC and VLPFC are activated during the valuation process. For decisions under risk, risk attitude is the crucial metric that is assumed to influence the value of a choice alternative. Up to now, only few studies tried to identify neural representations of risk attitude; so far correlated regions have been found in IOFC, mOFC, PCC, and VLPFC.

The usual technique used in neuroscience to evaluate the fMRI data is the GLM approach. This approach has some limitations that are crucial for the identification of brain regions associated with risk attitude. GLM focuses on the changes in the mean BOLD signal and hereby neglects the information in the variance of the signal. Further, GLM is a model-based technique that is able to detect only pre-defined effects.

In this paper, we applied a novel nonparametric statistical model to analyse fMRI data from an experiment associated with risky decisions. Our panel dynamic semiparametric factor model (PDSFM) is a model-free, dimension reduction technique with a minimum number of model parameters. DSFM provides spatial maps common for all studied subjects and time-variant factor loadings which are specific for each individual. Both spatial maps and factor loadings are very easy to interpret. Statistical inference of the whole high-dimensional data set is based only on the low-dimensional time series (factor loadings). 
Applying the PDSFM, we analyzed an fMRI experiment with 17 subjects, each with 722 images of $91 \times 109 \times 91$ voxels. We identified 20 spatial factors $\widehat{m}_{l}$, six of them corresponding to mOFC and PC which were already found in decision making context. Other spatial factors were associated with brain areas playing roles in motor responses and visual perception which were also related to the experiment. Our identified 20 spatial factors, however, did not contain any activation regions previously reported by Mohr et al. (2010b) using the GLM approach. Only the PC was identified in a recent meta-analysis on neural representations of risk (Mohr, Biele, \& Heekeren, 2010a). Mohr and colleagues (2010b) identified neural representations of perceived risk in the anterior (AI) insula, neural representations of subjective value in medial prefrontal cortex (MPFC), dorsolateral prefrontal cortex (DLPFC), and ventrolateral prefrontal cortex (VLPFC), and neural representations of risk attitude in 1OFC and PCC. There are at least three possible reasons that could account for the different results. First, Mohr et al. (2010b) concentrated their analysis on a priori pre-specified regions which were expected to show correlations with risky decisions. Second, the results from Mohr et al. (2010b) were based on regressors for the decision trials, which capture only around $5 \%$ of the time series. And, finally, the regressors for perceived risk and subjective expected return model use very specific effects, namely the correlation between regressors and the BOLD response on a trial-by-trial basis. It is, therefore, very unlikely that the first 20 factors, identified in a PDSFM using only part of the data, capture the described very specific effects based on only $5 \%$ of the high-dimensional time series.

Further, we provided the statistical analysis of factor loadings corresponding to the spatial maps in mOFC and PC. We observed that the variability in the responses after the decision stimuli is significantly higher for weakly risk-averse individuals than for strongly risk-averse individuals. We used the variance of these stimuli responses as input for the classification algorithm. Very high classification rates (97\% and $75 \%$ ) were obtained with the SVM classifier by applying the double cross-validation algorithm. We classified the risk attitude of the subjects from the low-dimensional representation of the brain activities without knowing the subject's answers. Herewith we have shown that our PDSFM approach is able to detect the neural representations of risk attitude and to classify the weak and strong averse individuals by their time-dependent factor loadings.

In the literature, the group fMRI analysis is usually carried out by the mixed effects functional model (Wang, 1998 and Guo, 2002). Mumford and Poldrack (2007) describe the mixed effects model in their application of it to fMRI data. Compared to their works, there are several differences in our model. First, their works mainly focus on the univariate (or low-dimensional) time series, while we focus on the full high-dimensional time series. Specifically for the fMRI data analysis, Mumford and Poldrack (2007) perform the analysis based on some (pre-specified) voxel of special interest, while we try to find both the temporal dynamics and the regions of special interest (w.r.t. risk attitude in our example) directly based on the high-dimensional fMRI time series without any a priori knowledge. Second, as illustrated via a political opinion study discussed in Mumford and Poldrack (2007), the mixed effect model considers the case where subjects participating in the experiment are drawn from several subgroups with their own groupspecific effects. Contrary, we assume that the subjects participating in our experiment were drawn randomly from the population without considering any special group effects. Our work, as far as we know, is one of the first works on panel high-dimensional time-series analysis with multiple subjects. One possible way of extending it to the mixed effect situation is to add the group effect to the time. Our approach may be applied to a wide spectrum of similar studies and can serve as a useful model-free technique for fMRI analysis. 
Appendix A. Residual $\varepsilon_{t, j}^{i}$ Analysis
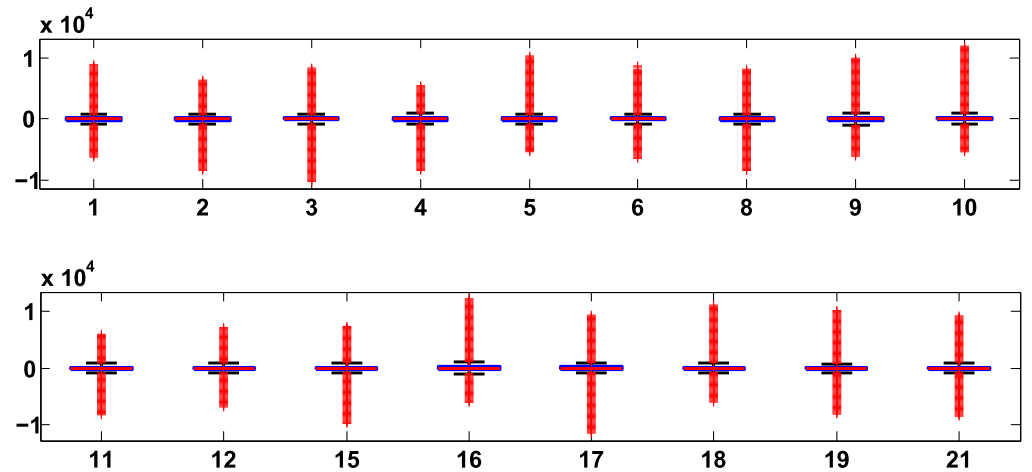

FIGURE 6.

Boxplots of random subsets (sample size $\left.3 \times 10^{7}\right)$ from $\varepsilon_{t, j}^{i}\left(4.3 \times 10^{9}\right.$ points) for all 17 analyzed subjects. Kurtosis exceeds 10 .
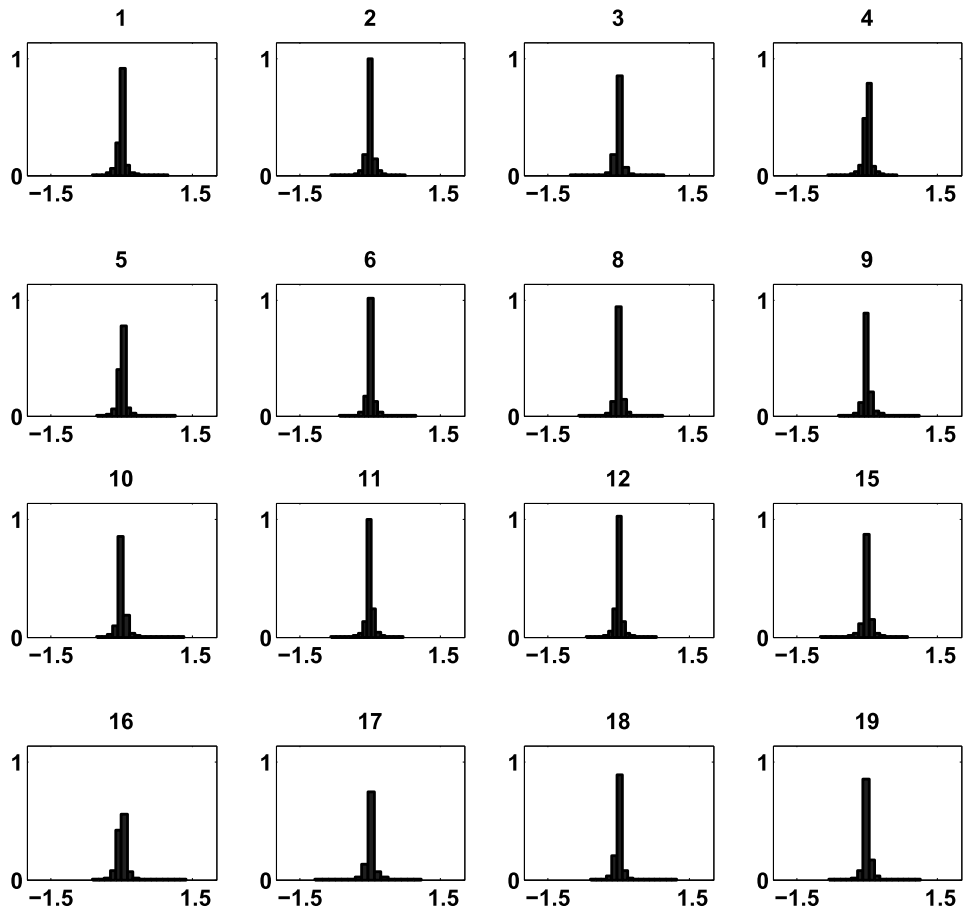

FIGURE 7.

Histograms of random subsets ( $\left.\operatorname{size} 3 \times 10^{7}\right)$ from $\varepsilon_{t, j}^{i}\left(4.3 \times 10^{9}\right.$ points) for subjects $i=1,2,3,4,5,6,8,9$ (upper panel) and $i=10,11,12,15,16,17,18,19$ (lower panel), respectively. Normality hypothesis (KS test) for standardized $\varepsilon_{t, j}^{i}$ rejected for all subjects at significance level $5 \%$. 

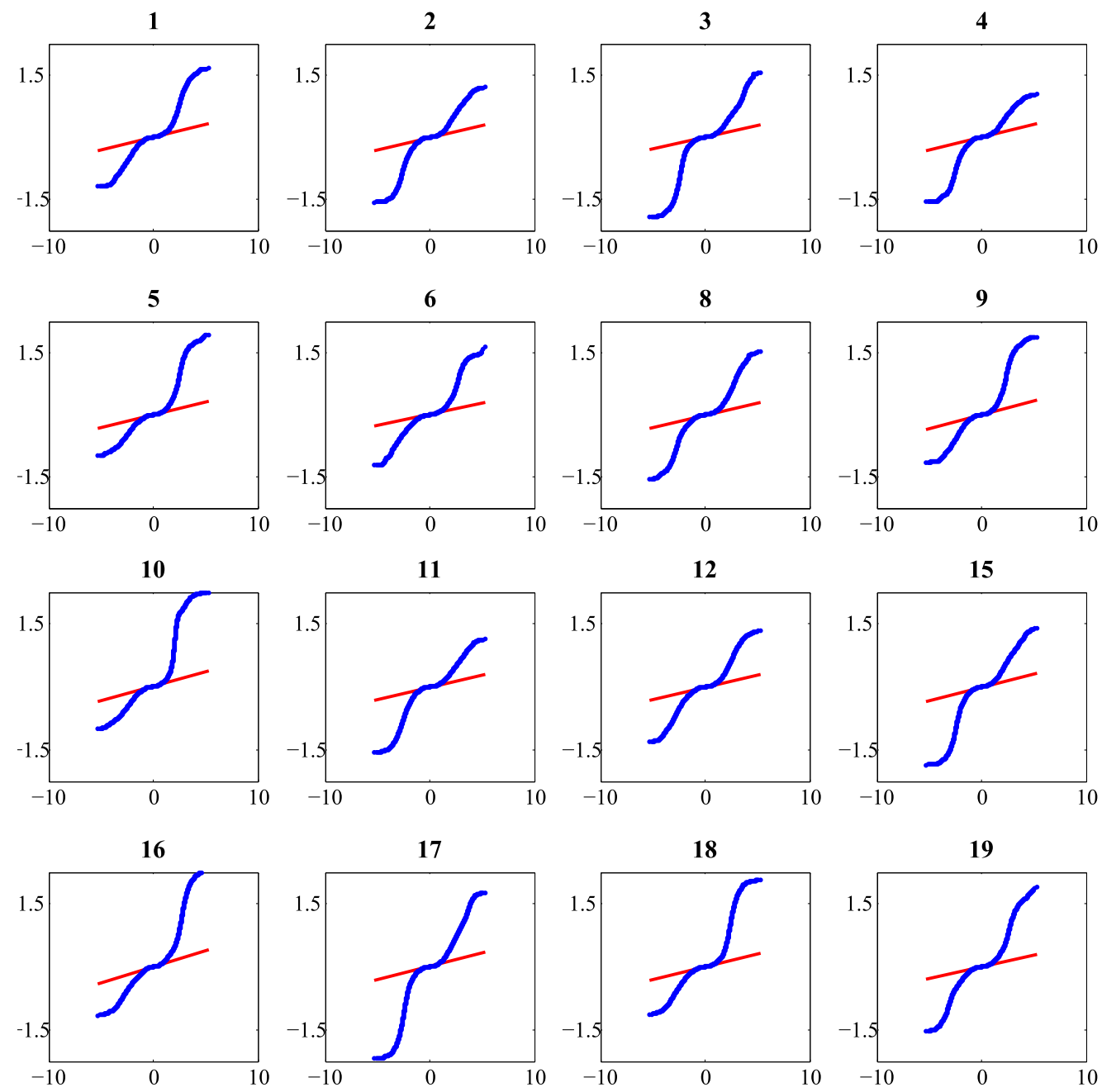

FIGURE 8.

QQ plots of random subsets (size $\left.3 \times 10^{7}\right)$ from $\varepsilon_{t, j}^{i}\left(4.3 \times 10^{9}\right.$ points) for subjects $i=1,2,3,4,5,6,8,9$ (upper panel) and $i=10,11,12,15,16,17,18,19$ (lower panel), respectively. (Color figure online.) 
Appendix B. Activity of Risk-Related Factors
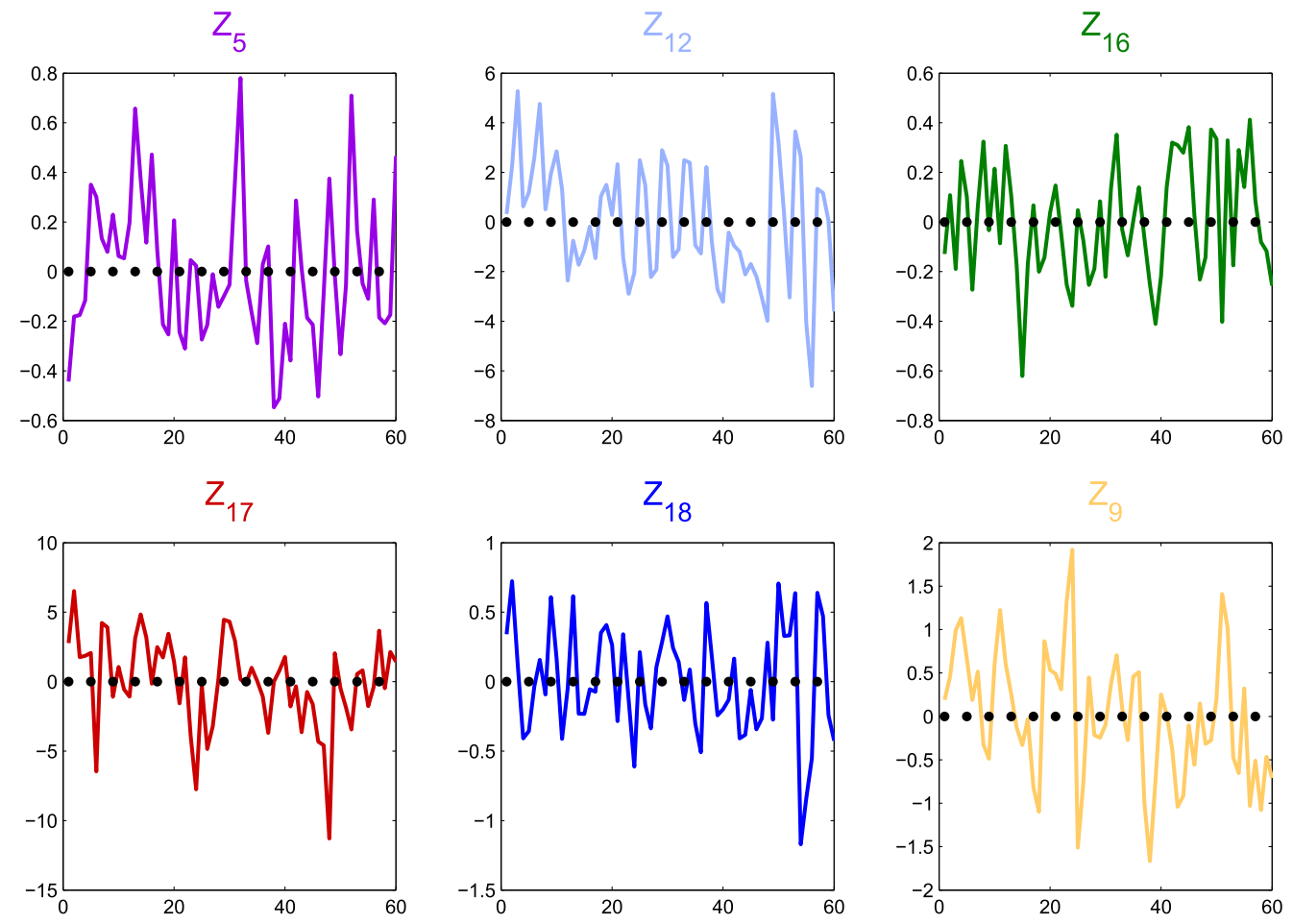

FIGURE 9.

Sum over all subjects of the normalized mean differences of the factor loadings $Z_{5}, Z_{12}, Z_{16}, Z_{16}, Z_{18}, Z_{9}$ corresponding to the risk-related factors, shown in the time point of decision task and the next three following time points. (Color figure online.) 
Appendix C. Reaction to Stimulus for Weakly and Strongly Risk-Averse Subjects
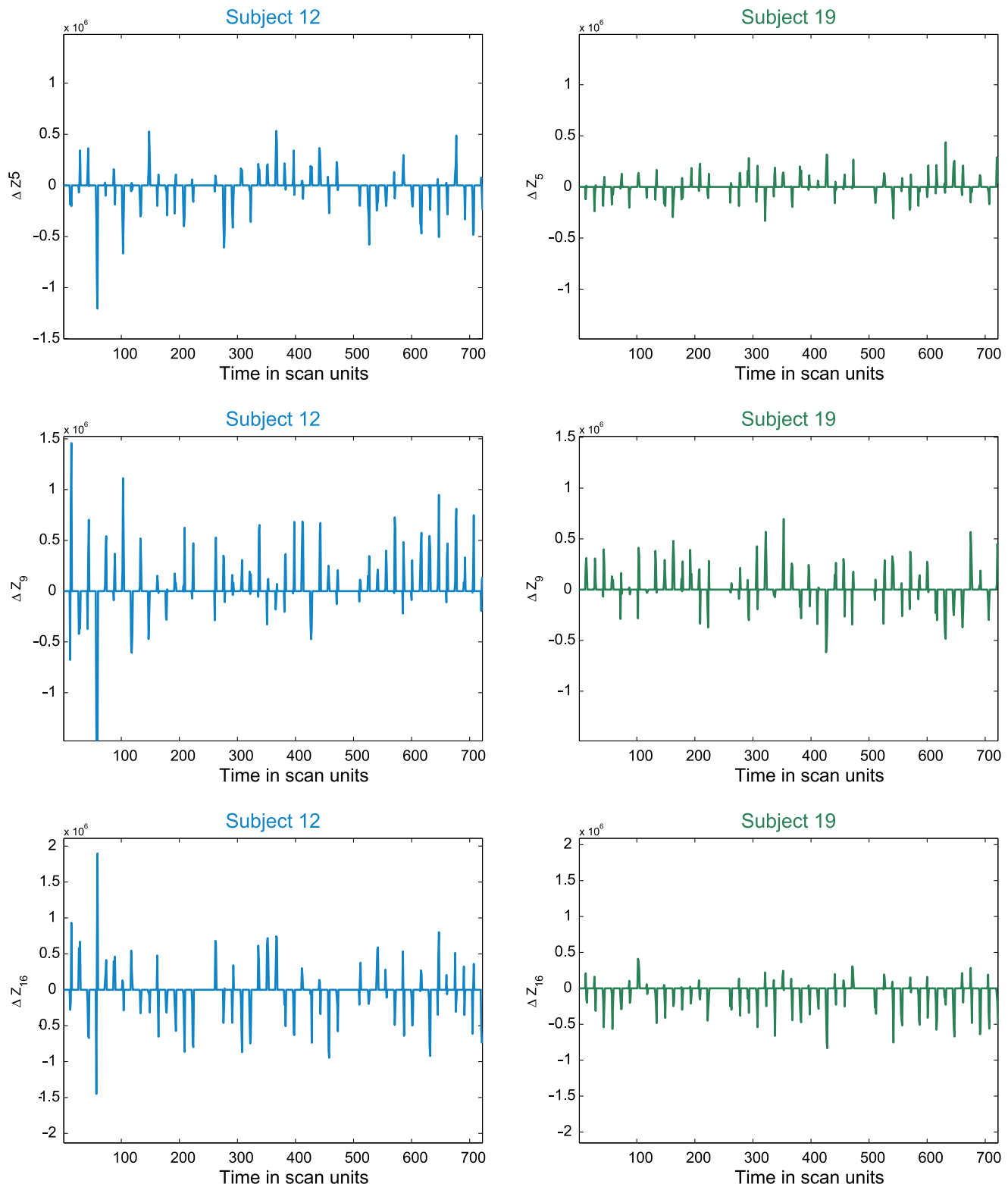

FIGURE 10.

Reaction to stimulus for factor loadings $\widehat{Z}_{t, l}, l=5,9,16,17$ (from the top) for weakly risk-averse Subject 12 (blue line, left panels) and strongly risk-averse Subject 19 (green line, right panels) during the whole experiment (45 stimuli). (Color figure online.) 

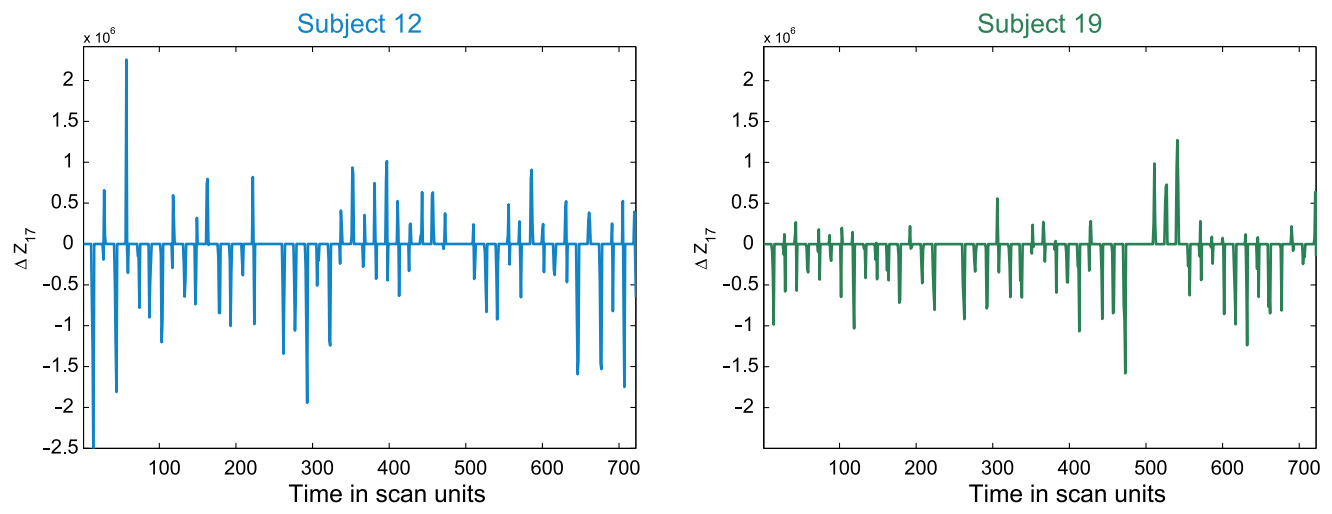

FIGURE 10.

(Continued.) 
Appendix D. Plots of Average Responses to the "Decision Under Risk" Stimulus
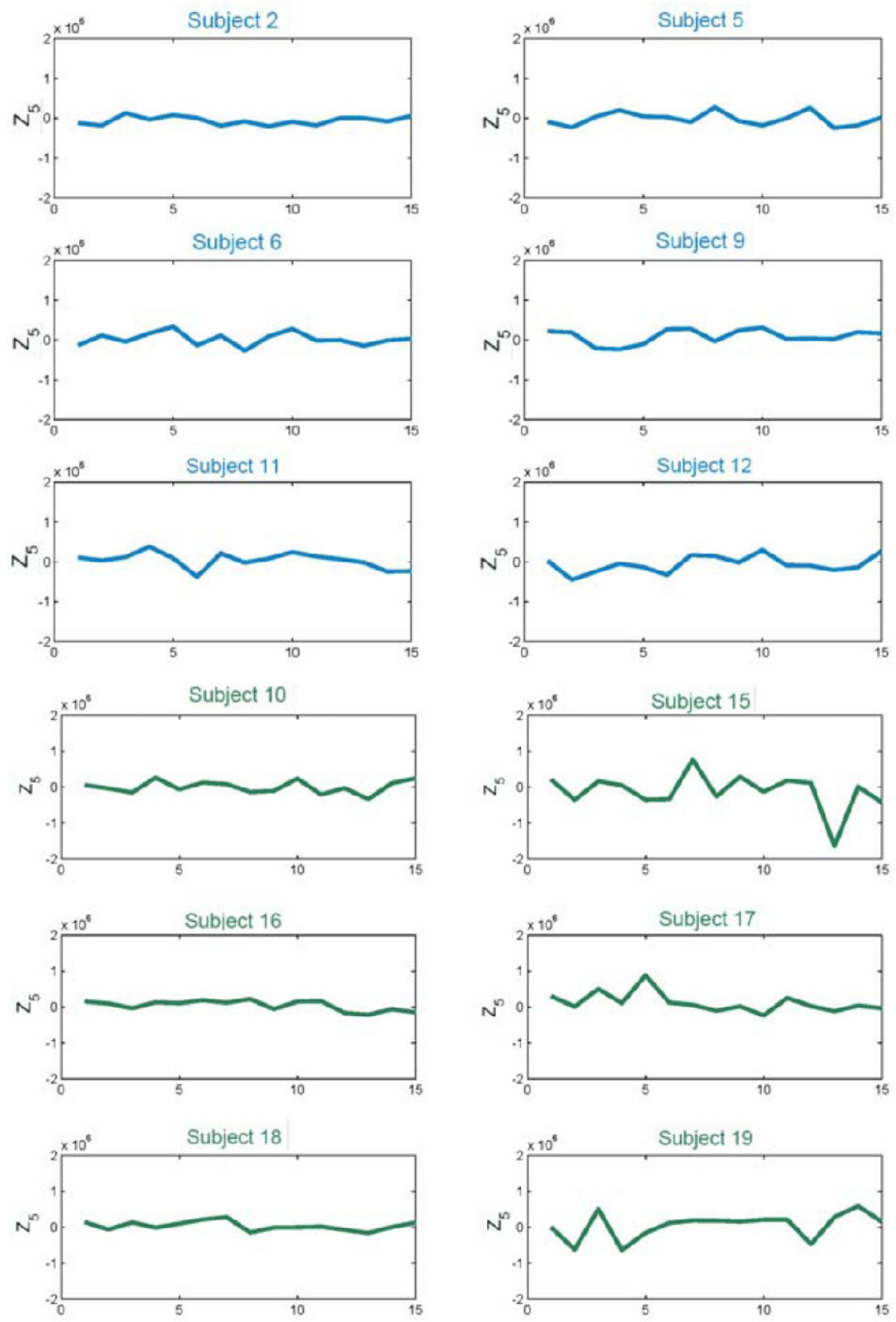

FIGURE 11.

Average responses to the "Decision under Risk" stimulus in factor loading $Z_{5}$ for the weakly risk-averse group (top, blue lines) and strongly risk-averse group (bottom, green lines). (Color figure online.) 

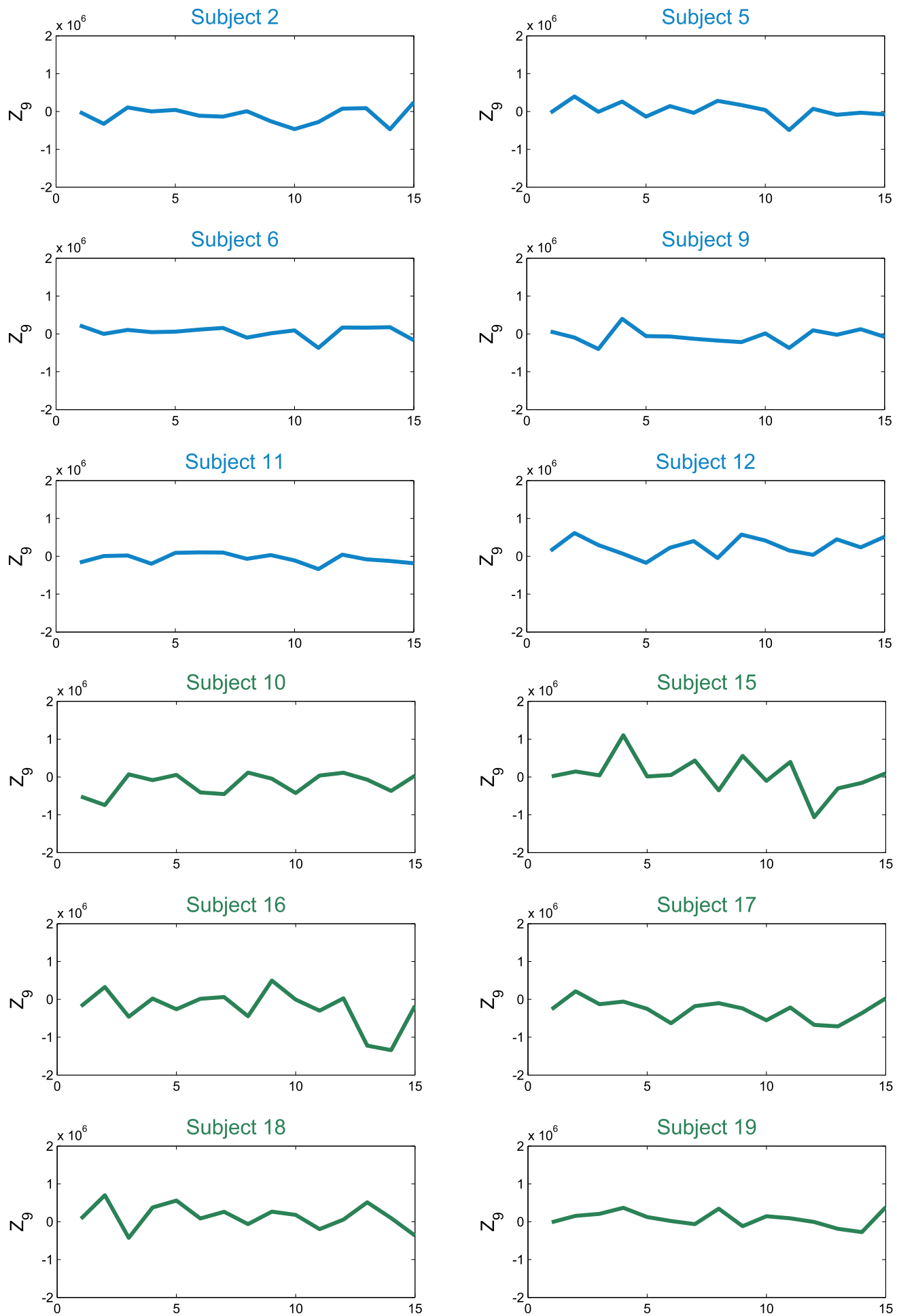

FIGURE 12.

Average responses to the "Decision under Risk" stimulus in factor loading $Z_{9}$ for the weakly risk-averse group (top, blue lines) and strongly risk-averse group (bottom, green lines). (Color figure online.) 

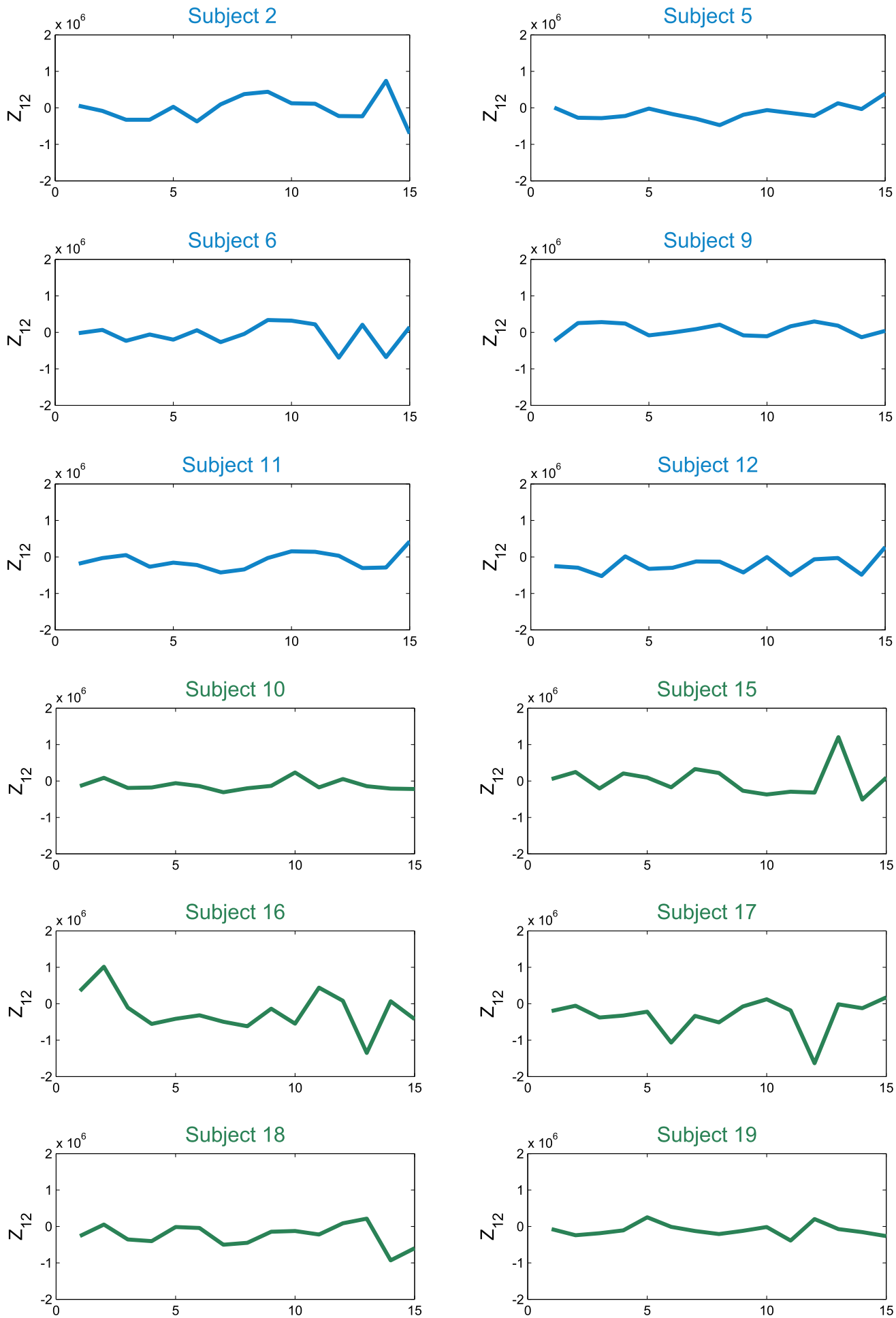

FIGURE 13.

Average responses to the "Decision under Risk" stimulus in factor loading $Z_{12}$ for the weakly risk-averse group (top, blue lines) and strongly risk-averse group (bottom, green lines). (Color figure online.) 

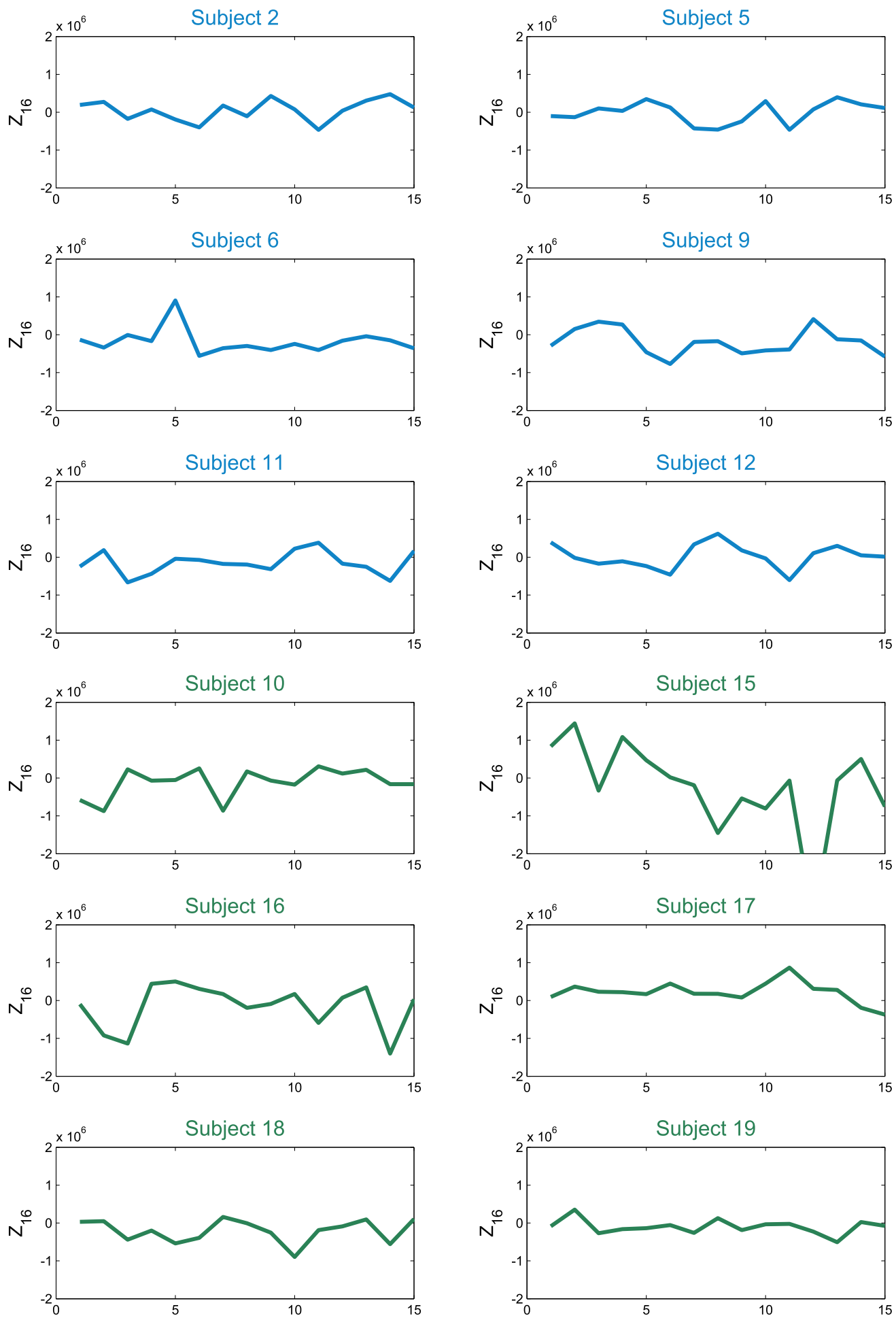

FIGURE 14.

Average responses to the "Decision under Risk" stimulus in factor loading $Z_{16}$ for the weakly risk-averse group (top, blue lines) and strongly risk-averse group (bottom, green lines). (Color figure online.) 

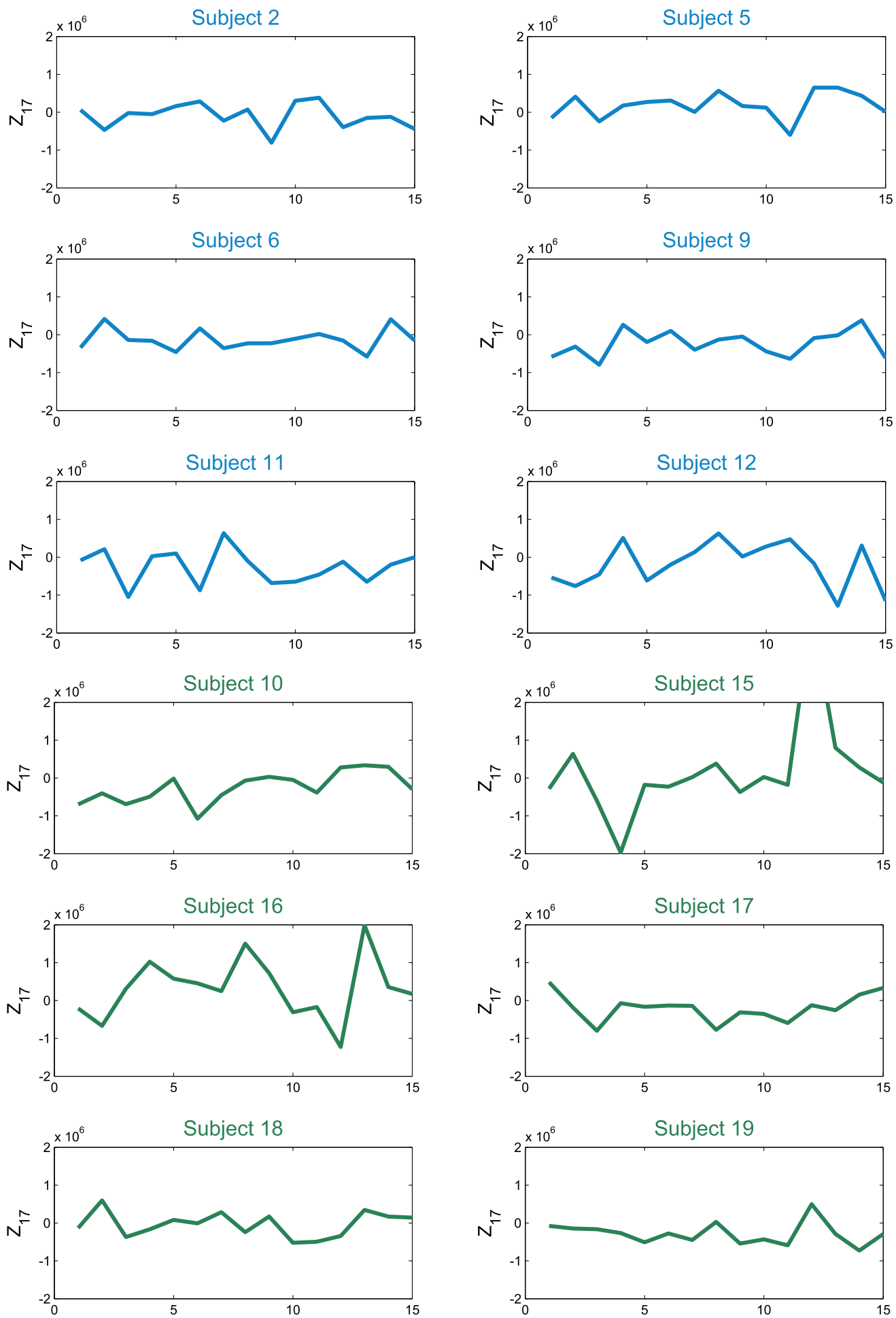

FIGURE 15.

Average responses to the "Decision under Risk" stimulus in factor loading $Z_{17}$ for the weakly risk-averse group (top, blue lines) and strongly risk-averse group (bottom, green lines). (Color figure online.) 

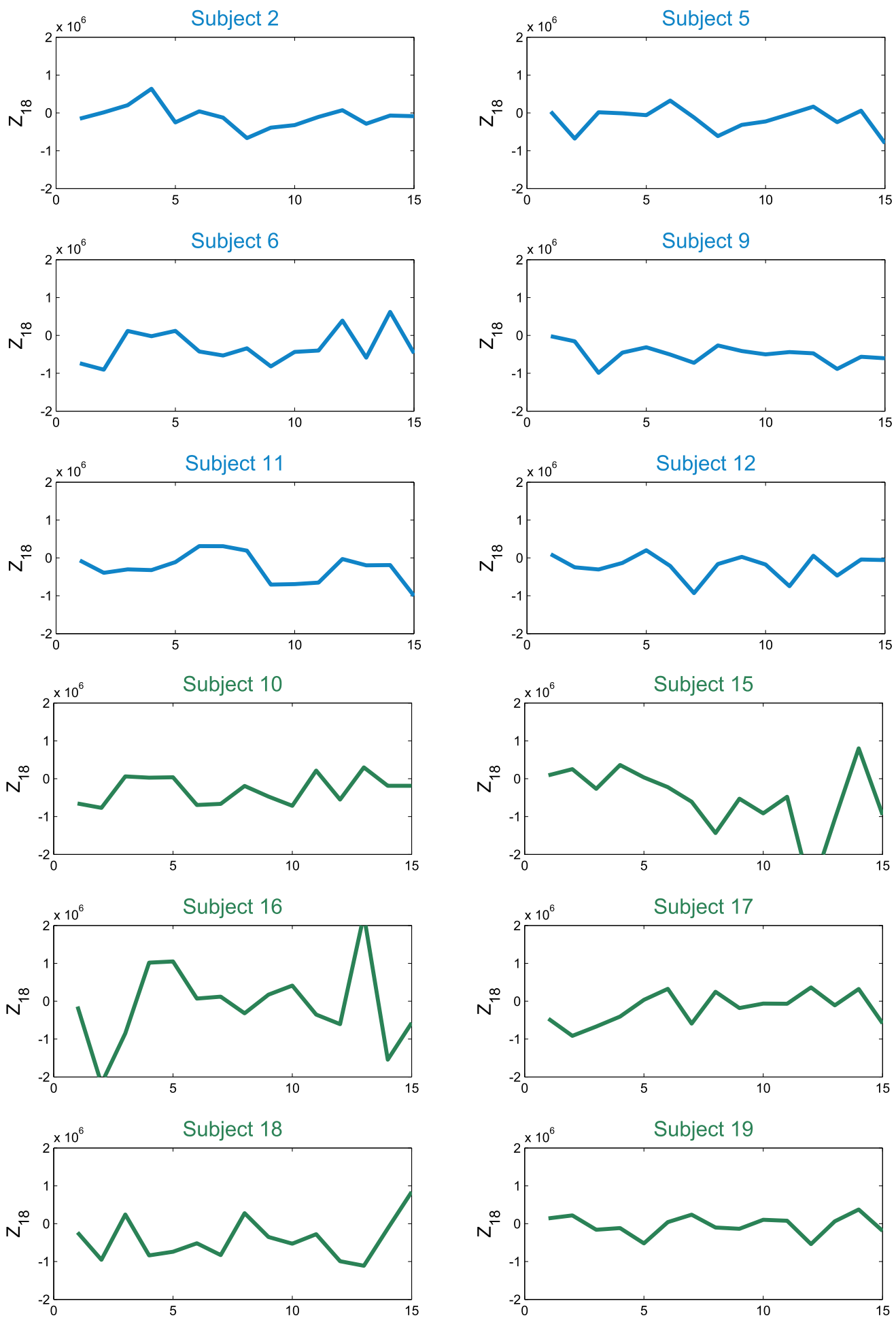

FIGURE 16.

Average responses to the "Decision under Risk" stimulus in factor loading $Z_{18}$ for the weakly risk-averse group (top, blue lines) and strongly risk-averse group (bottom, green lines). (Color figure online.) 
Beckmann, C., \& Smith, S. (2005). Tensorial extensions of independent component analysis for multisubject FMRI analysis. NeuroImage, 25, 294-311.

Behrmann, M., Geng, J., \& Shomstein, S. (2004). Parietal cortex and attention. Current Opinion in Neurobiology, 14, 212-217.

Cortes, C., \& Vapnik, V. (2005). The nature of statistical learning theory’. Machine Learning, 20, 273-297.

Guo, W. (2002). Functional mixed effects models. Biometrics, 58, 121-128.

Heekeren, H., Marrett, S., \& Ungerleider, L. (2008). The neural systems that mediate human perceptual decision making. Nature Reviews. Neuroscience, 9, 467-479.

Kable, J., \& Glimcher, P. (2007). The neural correlates of subjective value during intertemporal choice. Nature Neuroscience, 10, 1625-1633.

Mohr, P., Biele, G., \& Heekeren, H. (2010a). Neural processing of risk. Journal of Neuroscience, 30(19), 6613-6619.

Mohr, P., \& Nagel, I. (2010). Variability in brain activity as an individual difference measure in neuroscience? Journal of Neuroscience, 30, 7755-7757.

Mohr, P.N.C., Biele, G., Krugel, L.K., Li, S.-C., \& Heekeren, H.R. (2010b). Neural foundations of risk-return trade-off in investment decisions. NeuroImage, 49, 2556-2563.

Mumford, J.A., \& Poldrack, R.A. (2007). Modeling group fMRI data. Social, Cognitive, and Affective Neuroscience, 2, 251-257.

Park, B.U., Mammen, E., Wolfgang, H., \& Borak, S. (2009). Time series modelling with semiparametric factor dynamics. Journal of the American Statistical Association, 104(485), 284-298.

Plassmann, H., O’Doherty, J., \& Rangel, A. (2007). Orbitofrontal cortex encodes willingness to pay in everyday economic transactions. Journal of Neuroscience, 37, 9984-9988.

Rangel, A., Camerer, C., \& Montague, P. (2008). A framework for studying the neurobiology of value-based decision making. Nature Reviews. Neuroscience, 9, 545-556.

Samanez-Larkin, G., Kuhnen, C., Yoo, D., \& Knutson, B. (2010). Variability in nucleus accumbens activity mediates age-related suboptimal financial risk taking. Journal of Neuroscience, 30, 1426-1434.

Sarin, R., \& Weber, M. (1993). Risk-value models. European Journal of Operational Research, 70, 135-149.

Tobler, P., O’Doherty, J., Dolan, R., \& Schultz, W. (2007). Reward value coding distinct from risk attitude-related uncertainty coding in human reward systems. Journal of Neurophysiology, 97, 1621-1632.

Wang, Y. (1998). Mixed effects smoothing spline analysis of variance. Journal of the Royal Statistical Society, Series B, $60,159-174$.

Weber, E., \& Johnson, E. (2009a). Mindful judgment and decision making. Annual Review of Psychology, 60, 53-85.

Weber, E., \& Johnson, E. (2009b). Neuroeconomics. Decision making and the brain (pp. 127-144). London: Elsevier. Ch. Decisions under uncertainty: psychological, economic, and neuroeconomic explanations of risk preference.

Weber, E.U., \& Milliman, R. (1997). Perceived risk attitudes: relating risk perception to risky choices. Management Science, 43(2), 122-143.

Weber, E.U., Siebenmorgen, N., \& Weber, M. (2005). Communicating asset risk: how name recognition and the format of historic volatility information affect risk perception and investment decisions. Risk Analysis, 25(3), 597-609.

Manuscript Received: 30 OCT 2012

Final Version Received: 6 FEB 2013

Published Online Date: 20 JUL 2013 